\title{
COMMENTARY
}

\section{Pocket ultrasound devices for focused echocardiography}

\author{
Daniel De Backer* and David Fagnoul \\ See related research by Biais et al., http://ccforum.com/content/16/3/R82
}

\begin{abstract}
Pocket ultrasound devices have recently been developed and may be particularly useful for emergency assessment. These devices can be stored in a pocket but share only some technical features with conventional echocardiographic machines. Two-dimensional imaging and color flow mode are available, with possible adjustments of global gain and depth, but Doppler features are lacking. These devices are particularly fitted for focused echocardiography. In this issue, a trial compares a pocket ultrasound device with a conventional echocardiographic machine for focused echocardiography in patients admitted to the emergency department. This commentary will put these findings into perspective.
\end{abstract}

Pocket ultrasound devices [1] appear particularly attractive but is this technology ready for use in emergency settings or intensive care units? Echocardiography is frequently used for hemodynamic evaluation in patients with circulatory or respiratory failure [2] as it is possible to use it to evaluate left and right ventricular function, preload, preload responsiveness, and pulmonary artery pressures. It allows not only the determination of the source of shock but also evaluation of filling pressures and volumes, and systolic and diastolic heart function. However, this approach may be time consuming and, more importantly, requires skills that can be achieved only after dedicated training and many supervised examinations [1,3]. A focused approach to echocardiography can nevertheless be obtained by most, including real beginners [4]. This approach allows the rapid evaluation of the main alterations contributing to hemodynamic instability and thus the orientation of therapy. Focused

*Correspondence: ddebacke@ulb.ac.be

Department of Intensive Care, Erasme University Hospital, Université Libre de Bruxelles, B-1070 Brussels, Belgium echocardiography consists of eyeball evaluation of left ventricular systolic function and evaluation of right ventricular enlargement, evaluation of preload responsiveness, and detection of cardiac tamponade [3,5]. This can be done semi-quantitatively by categorizing function or dilation as normal, moderately altered, or markedly altered [6]. This simplified echocardiographic approach can be performed by many operators, as minimal skills are required. Indeed, after a 3 hour training course and 5 hours of hands-on training, beginners can acquire enough skills to adequately estimate the different components of focused echocardiography [4] and to identify most life-threatening hemodynamic alterations. This approach and skill requirement are now recognized by scientific societies [3] and implemented in core ultrasound training.

However, one piece was still missing from the puzzle of focused echocardiography: until recently, focused echocardiography was often performed with relatively large echo machines, which are not really suitable for focused echocardiography as they are heavy to mobilize, use a large part of the limited space available at the bedside, and, more importantly, often have a long setup time, which may be an issue when emergency evaluation is required. Over the past years, several companies have developed portable ultrasound machines that have greatly improved the possibility of rapidly moving them for emergency assessment. Additionally, pocket ultrasound devices have now been developed. These devices can be stored in a pocket but share only some technical features with the larger devices. Two-dimensional imaging and color flow mode are available, with possible adjustments of global gain and depth. However, more advanced features, such as time-motion mode, and pulsed and continuous Doppler, are not available. The information provided by these devices theoretically fits the scope of focused echocardiography.

Although pocket ultrasound devices provide excellent quality images in selected populations, the crucial question of whether these devices can be used in an unselected population in an emergency setting remained unanswered. In this issue, Biais and colleagues [1] 
evaluate whether the findings observed during focused echocardiography with a portable ultrasound device reliably reflect those that can be obtained with a classical echocardiography device. In this elegant trial, the authors investigated 151 patients requiring evaluation with focused echocardiography in an emergency department. In each case two intensivists independently, and blind to the other examination, conducted a focused echocardiographic assessment using either the pocket ultrasound device or a classical echocardiography device. Parasternal, apical, and subcostal views were obtained to evaluate global cardiac systolic function, ventricular enlargement or hypertrophy, presence of a pericardial effusion and size and the respiratory changes of the inferior vena cava diameter. All measurements were qualitative except for ejection fraction, which was visually determined. In no case did the pocket device technology fail to provide a definitive diagnosis; when a diagnosis could not be obtained with one device it was also the case with the other device. With the portable ultrasound device it was possible to adequately evaluate the different items with good to excellent agreement. In addition, ejection fraction was correctly evaluated with no bias and narrow limits of agreement (13.5\%).

The authors nicely show that this portable ultrasound device is perfectly suited for use in the emergency department. It would also fit perfectly in an ambulance. But would this kind of device also be useful in the intensive care unit? Obviously, image quality was sufficient for focused echocardiography, but this does not mean that image quality was similar between the two devices and acquisition of good images may be more difficult in critically ill patients, and in particular in mechanically ventilated patients. In addition, portable ultrasound devices may not be suitable for more advanced echocardiographic studies; for example, measurements of cardiac output and filling pressures are not feasible in the absence of Doppler technologies. But to what extent is this really a limiting factor? First, one may guess that improvements in technologies will allow some of these limitations to be overcome in the future; in particular, image acquisition is likely to improve. Doppler techniques could also be implemented, allowing cardiac output monitoring, but this is not a high priority. In many patients, other hemodynamic monitoring devices are used to provide cardiac output measurements. And, of course, conventional high-tech ultrasound devices will still have a place for more precise assessment.

In conclusion, pocket ultrasound devices are now ready for wide use in emergency department and ambulance settings for focused echocardiography.

\section{Competing interests}

The authors declare that they have no competing interests.

Published: 29 June 2012

\section{References}

1. Biais M, Carrie C, Delaunay F, Morel N, Revel P, Janvier G: Evaluation of a new pocket echoscopic device for focused cardiac ultrasonography in an emergency setting. Crit Care 2012, 16:R82.

2. De Backer D, Cholley BP, Slama M, Vieillard-Baron A, Vignon P: Hemodynamic Monitoring Using Echocardiography in the Critically III. Heidelberg, Dordrecht, London, New York: Springer; 2011.

3. Mayo PH, Beaulieu Y, Doelken P, Feller-Kopman D, Harrod C, Kaplan A, Oropello J, Vieillard-Baron A, Axler O, Lichtenstein D, Maury E, Slama M, Vignon P: American College of Chest Physicians/La Societe de Reanimation de Langue Francaise statement on competence in critical care ultrasonography. Chest 2009, 135:1050-1060.

4. Vignon P, Dugard A, Abraham J, Belcour D, Gondran G, Pepino F, Marin B, Francois B, Gastinne H: Focused training for goal-oriented hand-held echocardiography performed by noncardiologist residents in the intensive care unit. Intensive Care Med 2007, 33:1795-1799.

5. Labovitz AJ, Noble VE, Bierig M, Goldstein SA, Jones R, Kort S, Porter TR, Spencer KT, Tayal VS, Wei K: Focused cardiac ultrasound in the emergent setting: a consensus statement of the American Society of Echocardiography and American College of Emergency Physicians. J Am Soc Echocardiogr 2010, 23:1225-1230.

6. Vieillard-Baron A, Charron C, Chergui K, Peyrouset O, Jardin F: Bedside echocardiographic evaluation of hemodynamics in sepsis: is a qualitative evaluation sufficient? Intensive Care Med 2006, 32:1547-1552.

doi:10.1186/cc11386

Cite this article as: De Backer D, Fagnoul D: Pocket ultrasound devices for focused echocardiography. Critical Care 2012, 16:134. 